\title{
NECESIDADES DE INVESTIGACIÓN PARA EL DIAGNÓSTICO DE ANEMIA EN POBLACIONES DE ALTURA
}

\author{
Gustavo F. Gonzales ${ }^{1,2, a, b}$, Diego Fano ${ }^{1, c}$, Cinthya Vásquez-Velásquez ${ }^{1, c}$
}

\begin{abstract}
RESUMEN
Tanto la deficiencia como la sobrecarga de hierro son situaciones que ponen en riesgo la salud y la vida de las personas, por lo que es importante mantener su homeostasis. Como la hemoglobina contiene $70 \%$ del hierro del organismo, la OMS recomienda su medición para determinar la prevalencia de anemia por deficiencia de hierro (ID), a pesar que ellos mismos reconocen que la anemia no es específica de ID. Como la hemoglobina aumenta con la altitud de residencia, la OMS recomienda corregir el punto de corte para definir anemia en la altura. Una objeción a esta corrección es que el aumento de la hemoglobina en la altura no es universal ni aumenta de manera lineal. Además, las poblaciones de mayor antigüedad generacional tienen menos hemoglobina que las más recientes. En infantes, niños, gestantes y adultos, la prevalencia de anemia usando hemoglobina corregida es 3-5 veces mayor que usando marcadores del estatus de hierro. Los programas estatales buscan combatir la anemia mediante la suplementación de hierro; no obstante, resultan ineficaces, especialmente en las poblaciones de altura. Entonces, ¿hay deficiencia de hierro en la altura? Los niveles de hepcidina sérica, hormona que regula la disponibilidad de hierro, son similares a los de nivel del mar indicando que en la altura no hay deficiencia de hierro. Un problema adicional al corregir la hemoglobina por la altura, es que las prevalencias de eritrocitosis disminuyen. En conclusión, la corrección del punto de corte de la hemoglobina en la altura para determinar deficiencia de hierro es inadecuada.
\end{abstract}

Palabras clave: Anemia, Deficiencia de hierro, Factor de corrección, Ferritinas (fuente: DeCS BIREME).

\section{DIAGNOSIS OF ANEMIA IN POPULATIONS AT HIGH ALTITUDES}

\begin{abstract}
Iron deficiency and overload are risk factors for numerous poor health outcomes, and thus the maintenance of iron homeostasis is vital. Considering that hemoglobin contains $70 \%$ of the total iron in the body, the World Health Organization (WHO) recommends the measurement of iron levels to calculate the rate of iron deficiency anemia (IDA), although WHO recognizes that IDA is not the only cause of anemia. As hemoglobin increases with altitude, WHO recommends correcting the cut-off point to define anemia at high altitudes. An objection to this correction is that the increase in hemoglobin at high altitudes is not universal and is not linear. In addition, individuals in older age groups have lower hemoglobin levels than those in younger age groups. In infants, children, pregnant women, and adults, the prevalence of anemia using corrected hemoglobin is 3-5 times higher than that using markers of iron status. State programs seek to control anemia by means of iron supplementation. However, these programs are ineffective, particularly for high-altitude populations. Therefore, the occurrence of iron deficiency at high altitudes is controversial. The serum levels of the hormone hepcidin, which regulates iron availability, are similar in individuals at high altitudes to those of individuals at sea level, indicating that iron deficiency does not occur at high altitudes. An additional problem when correcting hemoglobin at high altitudes is that the frequency of erythrocytosis is decreased. In conclusion, the correction of the cut-off point of hemoglobin at high altitudes to determine iron deficiency is inadequate.
\end{abstract}

Key words: Anemia, Iron deficiency, Correction factor, Ferritins (source: MeSH NLM).

\section{INTRODUCCIÓN}

El Perú tiene treinta millones de habitantes de los cuales diez viven por encima de los 2000 metros de altitud. La vida en las alturas se caracteriza por un aumento en los niveles de hemoglobina como respuesta a la hipoxia ambiental.
Aunque por muchos años se tomó como cierto que conforme aumenta la altura se observa un aumento en la hemoglobina, en la actualidad esto ya no es aceptado. En efecto, los tibetanos muestran un umbral a partir del cual se observa un aumento de la hemoglobina recién a partir de los $3800 \mathrm{~m}$ de altitud ${ }^{(1)}$. Asimismo, los tibetanos y etíopes

\footnotetext{
Departamento de Ciencias Biológicas y Fisiológicas, Facultad de Ciencias y Filosofía, Universidad Peruana Cayetano Heredia. Lima, Perú

Instituto de Investigaciones de la Altura, Universidad Peruana Cayetano Heredia. Lima, Perú

a Doctor en Ciencias; ${ }^{\mathrm{b}}$ doctor en Medicina; ${ }^{\mathrm{C}}$ licenciado

Recibido: 29/10/2017 Aprobado: 30/11/2017 En línea: 07/12/2017
}

Citar como: Gonzales GF, Fano D, Vásquez-Velásquez C. Necesidades de investigación para el diagnóstico de anemia en poblaciones de altura. Rev Peru Med Exp Salud Publica. 2017;34(4):699-708. doi: 10.17843/rpmesp.2017.344.3208 
tienen menos concentración de hemoglobina que los Han en el Himalaya y que los andinos del Perú y Bolivia, cuando se comparan poblaciones viviendo a una misma altitud (1). En Perú, los habitantes en los Andes del sur tienen menor hemoglobina que aquellos en los Andes centrales. Estas diferencias se han tratado de explicar cómo diferencias en el tiempo multigeneracional de residencia en la altura ${ }^{(2)}$. En efecto, las poblaciones de los Andes del sur con mayor proporción aimara (>50\%) tienen mayor peso al nacer que aquellos con menos de $50 \%$ de proporción aimara viviendo en las mismas zonas altitudinales ${ }^{(3)}$. Igualmente, en Puno se ha observado que las gestantes aimaras de zonas rurales tienen una menor tasa de muerte fetal tardía que las rurales quechuas, que las urbanas o que los migrantes de zonas bajas ${ }^{(4)}$.

El peso al nacer es menor conforme aumenta la altitud de residencia en los Andes centrales. Este patrón es diferente en los Andes del sur del país, donde los aimaras de Puno (3800 $\mathrm{m}$ ) tienen un mayor peso al nacer que en los Andes centrales con similar y aun a menor altitud, como Huancayo $(3280 \mathrm{~m})^{(5)}$. Este menor peso al nacer ocurre sin cambios en la edad gestacional ${ }^{(6)}$. Una situación similar se observa entre tibetanos que tienen viviendo en el Himalaya casi 25 000 años y los de la etnia china Han con tan solo 60 años de antigüedad generacional. Los tibetanos muestran mayor peso al nacer asociado a menores valores de hemoglobina materna ${ }^{(7,8)}$. En las grandes alturas de Bolivia, las gestantes de ascendencia Andina tienen niños con mayor peso al nacer que aquellas consideradas mestizas y aquellas con ascendencia europea ${ }^{\left({ }^{(9)}\right.}$. Se explica el mayor peso al nacer como un efecto a un mayor flujo útero placentario en las poblaciones adaptadas a la altura ${ }^{(10)}$.

En Perú, la muerte fetal tardía es 4,8 veces más frecuente en la altura (>3000 metros sobre el nivel del mar) que a baja altitud. Este efecto se observa a pesar de controlar por factores socioeconómicos, demográficos y biológicos, control prenatal, y factores fetales. Sin embargo, los habitantes de los Andes del sur tienen una menor tasa de muerte fetal tardía, debido a su mayor antigüedad generacional ${ }^{(1)}$. Estos pobladores del sur muestran valores de hemoglobina materna más bajos debidos a un proceso de adaptación a la altura y no por ser anémicos ${ }^{(2,12)}$.

Según el Centro Nacional de Alimentación y Nutrición (CENAN-INS), la prevalencia de desnutrición crónica de niños menores de 5 años es más alta en Huancavelica (3600 m en Andes centrales) con 30,6\%, en tanto Puno (3800 m en Andes del sur) con $14,6 \%$, se encuentra por debajo de la media nacional (15,3\%); sin embargo, cuando se evalúa la anemia en menores de 3 años, la mayor prevalencia se observa en Puno con 75,2\%. Un análisis de regresión lineal entre poblaciones ubicadas en la costa y selva muestra una relación lineal directa entre la prevalencia de desnutrición crónica y prevalencia de anemia (Figura 1A). Cuando se

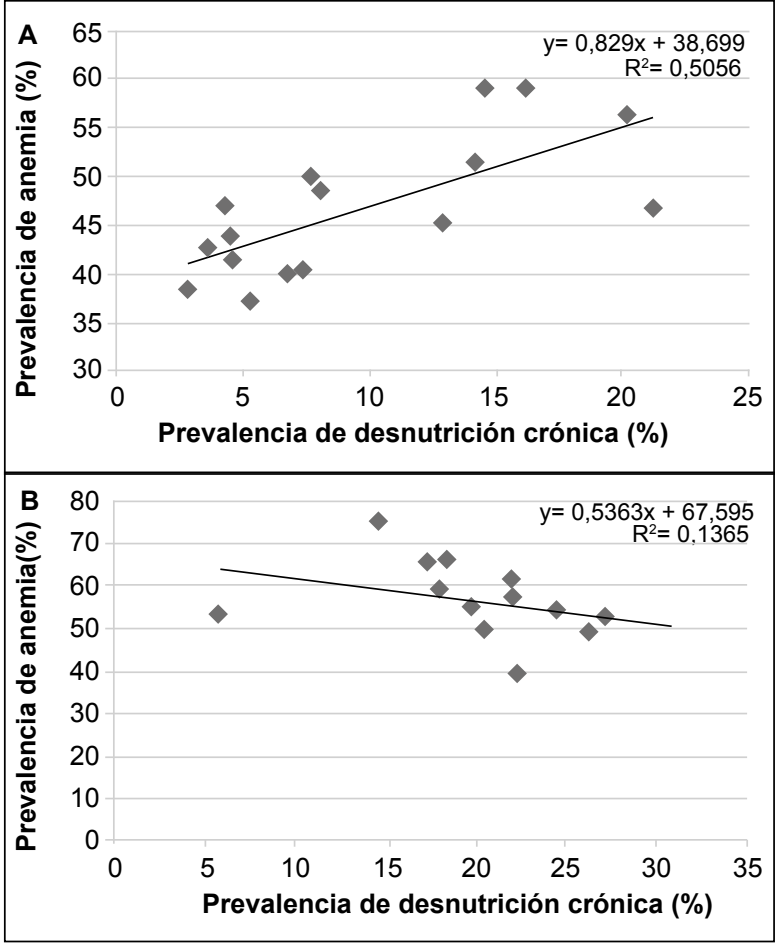

Figura 1. A) Correlación entre la prevalencia de desnutrición crónica en niños de 5 años y prevalencia de anemia en niños de 3 años de la región costa y selva (baja altitud), considerando los siguientes lugares evaluados: La Libertad, Ucayali, Loreto, Sullana, Madre de Dios, Lima, Moquegua, Piura, Lambayeque, Lima Sur, Lima ciudad, Lima Este, Tumbes, Ica, Tacna y Callao. B) Correlación entre la prevalencia de desnutrición crónica en niños de 5 años y prevalencia de anemia en niños de 3 años de la región sierra (gran altitud), considerando los siguientes lugares evaluados: Puno, Cusco, Pasco, Ayacucho, Junín, Apurímac, Ancash, Andahuaylas, Arequipa, Cajamarca, Huancavelica, Huánuco, Chota, Cutervo (Fuente: Instituto Nacional de Salud, Centro Nacional de Alimentación y Nutrición) ${ }^{(52)}$.

analiza solo los departamentos ubicados en la sierra no se observa esta asociación (Figura 1B). Esta discrepancia parece deberse al criterio usado para definir anemia en la altura ${ }^{(12,13)}$.

\section{REQUERIMIENTO DE HIERRO}

El hierro es importante para el funcionamiento y supervivencia de las células de casi todos los organismos vivientes, por ello es necesario evitar su deficiencia. Sin embargo, su exceso genera estrés oxidativo, que es también dañino para la función y supervivencia de las células y tejidos. Por ello es importante mantener la homeostasis del hierro (Figura 2) ${ }^{(14,15)}$. Uno de los mecanismos que utiliza el organismo para mantener la homeostasis del hierro es evitar su excreción; así, las pérdidas por descamación de células entéricas y otras son del nivel de $1 \mathrm{mg}$ diario, que es lo que habría que reponer con la dieta.

Si la pérdida de hierro es mayor o si la necesidad aumenta como en el embarazo (donde se requiere un gramo 


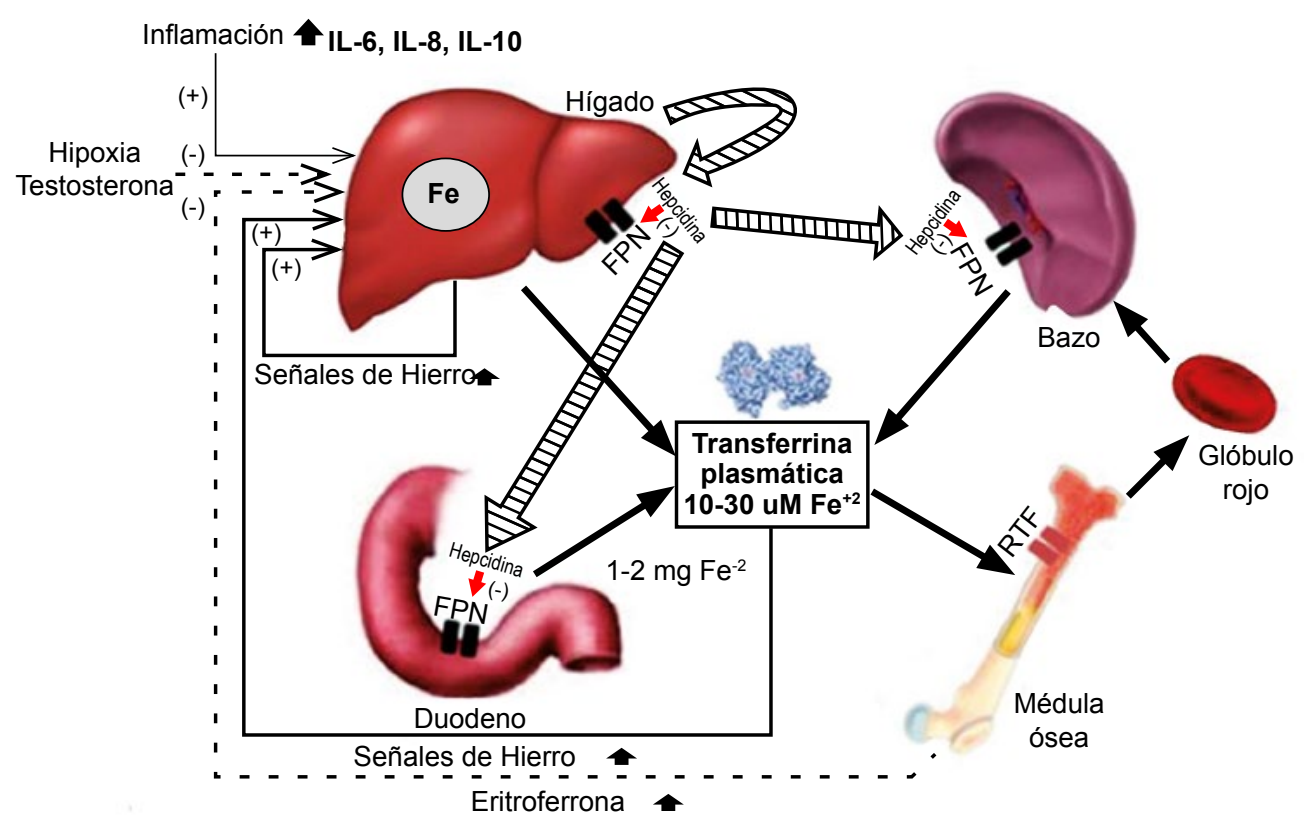

Figura 2. Homeostasis del hierro: rol de la hepcidina, eritroferrona, transferrina y receptor de transferrina. La inflamación, el exceso de hierro y el aumento de ferritina aumentan los niveles de hepcidina sérica para disminuir la disponibilidad de hierro. La eritroferrona, hipoxia aguda, bajo contenido corporal de hierro, y testosterona disminuyen los niveles de hepcidina sérica para favorecer la disponibilidad de hierro.

adicional para el feto y la placenta), el organismo necesitará absorber mayor cantidad de hierro (Figura 3). Esta absorción es regulada por la hepcidina, hormona peptídica producida en el hígado, considerada la principal reguladora de la homeostasis del hierro ${ }^{(16)}$. La hepcidina descubierta el 2000 tiene propiedades antibacterianas y por ello se le conoció como péptido antimicrobiano hepcidina (HAMP) ${ }^{(17)}$. La actividad antimicrobiana es debida a su capacidad de disminuir la disponibilidad de hierro a los microorganismos evitando su supervivencia.

La hepcidina liga al receptor ferroportina en el duodeno, lugar donde se absorbe el hierro, y en las células en las cuales el hierro está almacenado como en el hígado, macrófagos, entre otros. Al unirse la hepcidina a la ferroportina, este transportador de hierro es internalizado y degradado evitando el ingreso de hierro hacia la circulación. Un aumento en el nivel de hepcidina reducirá la disponibilidad de hierro al organismo. Por el contrario, una reducción de hepcidina aumentará la disponibilidad de hierro ${ }^{(18)}$. Una vez que se ha obtenido el valor de adulto, tanto para el contenido de hierro como para la hepcidina, los niveles de hepcidina van a modificarse según el requerimiento de hierro que tenga el organismo que, a su vez, dependerá de la disponibilidad de hierro almacenado. Los niveles en suero de hepcidina disminuyen cuando la demanda de hierro es alta como sería el caso si los nativos de la altura fueran deficientes de hierro.
Alrededor del $70 \%$ del hierro se localiza en la hemoglobina y casi un gramo se almacena en el hígado ligado a la ferritina. Por ello, se utiliza la medición de la hemoglobina como un marcador del hierro del organismo. Normalmente el varón adulto tiene $4 \mathrm{~g}$ y la mujer adulta $3 \mathrm{~g}$ de hierro en el organismo. Cada día se produce $20 \mathrm{~mL}$ de sangre, la cual contiene $6 \mathrm{~g}$ de hemoglobina y $20 \mathrm{mg}$ de hierro ${ }^{(19)}$.

Durante la exposición aguda a la altura hay una reducción en los niveles séricos de hepcidina, como respuesta a la hipoxia y no a una deficiencia de hierro ${ }^{(20)}$. En los nativos de la altura, la situación es diferente, como ha sido demostrado en etíopes, los valores basales de hepcidina en adultos son similares o mayores que a baja altitud ${ }^{(21)}$ lo que demuestra que son suficientes en hierro. Cuando se hace sangría en la altura, los niveles de hepcidina se reducen para aumentar la disponibilidad de hierro producida por la extracción de sangre ${ }^{(22)}$.

Las gestantes requieren un gramo adicional de hierro para el feto y la placenta. Por lo tanto, se requiere aumentar notablemente la absorción de hierro. Es por ello, que los niveles de hepcidina se reducen en el segundo trimestre de gestación ${ }^{(23)}$ con la finalidad de aumentar la absorción de hierro en el duodeno ${ }^{(24,25)}$.

En humanos, aproximadamente el $80 \%$ del hierro fetal se acumula en el tercer trimestre del embarazo, con una 


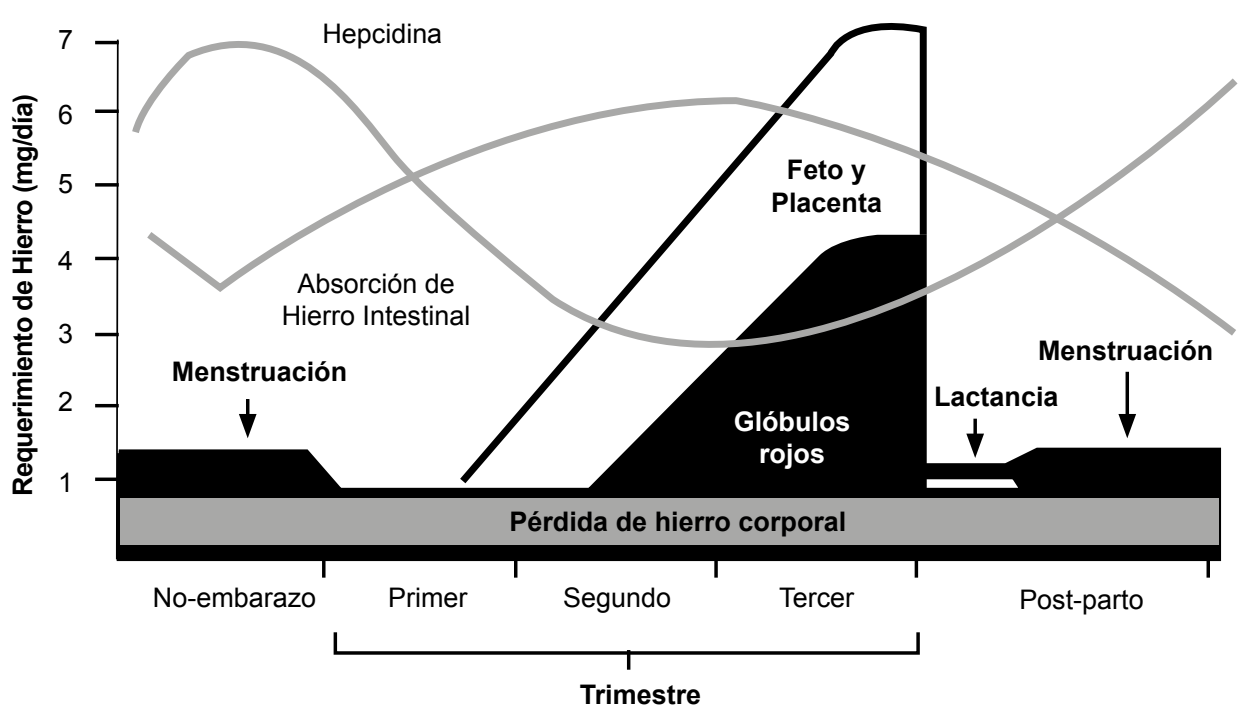

Figura 3. Requerimiento de hierro en el embarazo, fluctuaciones de hepcidina y absorción de hierro intestinal en los tres trimestres de gestación ${ }^{(53,54)}$.

velocidad máxima de transferencia estimada de $7 \mathrm{mg} / \mathrm{d}^{(26)}$. Esto ha cambiado el paradigma de a mayor requerimiento de hierro en el organismo, la cantidad de hierro consumido en la dieta debe aumentar. En efecto, la disminución de la hepcidina con el embarazo aumenta la absorción intestinal de hierro hasta en siete veces el valor pregestacional (Figuras 3, 4F).

Como la eritropoyesis aumenta en las gestantes, también aumenta el número de glóbulos rojos, lo cual aumentaría la viscosidad de la sangre y ello afectaría el flujo úteroplacentario, que, a su vez, reduciría la disponibilidad de nutrientes al feto. Para evitar esta situación, el organismo aumenta el volumen plasmático en una proporción mayor al aumento de la masa eritrocitaria y como resultado de esto se reduce la concentración de hemoglobina (hemodilución).

El feto crece en condiciones hipóxicas en el útero, por lo que al nacer tiene un valor elevado de hemoglobina, principalmente tipo $\mathrm{F}$. La hemoglobina $\mathrm{F}$ es destruida en las primeras semanas de vida y el hierro que contiene es reciclado para producir hemoglobina $\mathrm{A}$.

Con este hierro que tiene al nacimiento y con lo que recibe de la madre por la leche materna, se estima que el infante tendrá hierro suficiente por seis meses cuando ocurre el destete, a partir del cual el requerimiento de hierro será cubierto con la dieta. Durante la niñez el contenido de hierro corporal $(\mathrm{CHC})$ es bajo, y luego aumenta durante la pubertad donde llegan a los valores de adulto (cuatro gramos en el varón y tres gramos en la mujer) (Figura 4B, C). En respuesta a estos valores de adulto del $\mathrm{CHC}$, los niveles de hepcidina del adulto serán mayores en varones que en mujeres ${ }^{(27)}$. En la etapa adulta, los requerimientos de hierro a ser absorbidos en el duodeno dependerán de los niveles de hepcidina sérica.

La situación es diferente cuando hay un proceso inflamatorio. En ese caso, los niveles de hepcidina aumentan y la disponibilidad de hierro disminuye. Esto determina una deficiencia de hierro por enfermedad crónica inflamatoria que puede o no asociarse a anemia ${ }^{(28)}$. En la Figura 4D puede apreciarse como a medida que aumentan los niveles de interleukina 8 en gestantes, aumentan los niveles de hepcidina en suero. También la obesidad se asocia a un proceso inflamatorio y favorece un aumento en los niveles de hepcidina en suero.

En la altura se observa un aumento en la eritropoyesis ${ }^{(29)}$. Sin embargo, también hay una reducción en volumen plasmático ${ }^{(30)}$. No está claro si las personas con eritrocitosis excesiva tienen mayor eritropoyesis que las personas normales de la altura o si ellos tienen menor volumen plasmático (hemoconcentración). Además, las personas con mayor residencia multigeneracional en la altura como los tibetanos y los etíopes tienen menores valores de $\mathrm{Hb}$ que aquellos con menor tiempo de residencia multigeneracional, como ocurre con la etnia Han en el Himalaya.

Teniendo en cuenta, que la medición de $\mathrm{Hb}$ se usa como un marcador indirecto del estado de hierro, la situación en la altura se complica debido a que para definir anemia se debería tener una deficiencia global de hemoglobina (baja eritropoyesis) y no una expansión del volumen plasmático (hemodilución) ${ }^{(31)}$, como ocurre en la gestación. Todos estos hallazgos sugieren que la $\mathrm{Hb}$ no es un buen marcador del estado de hierro en el organismo. 


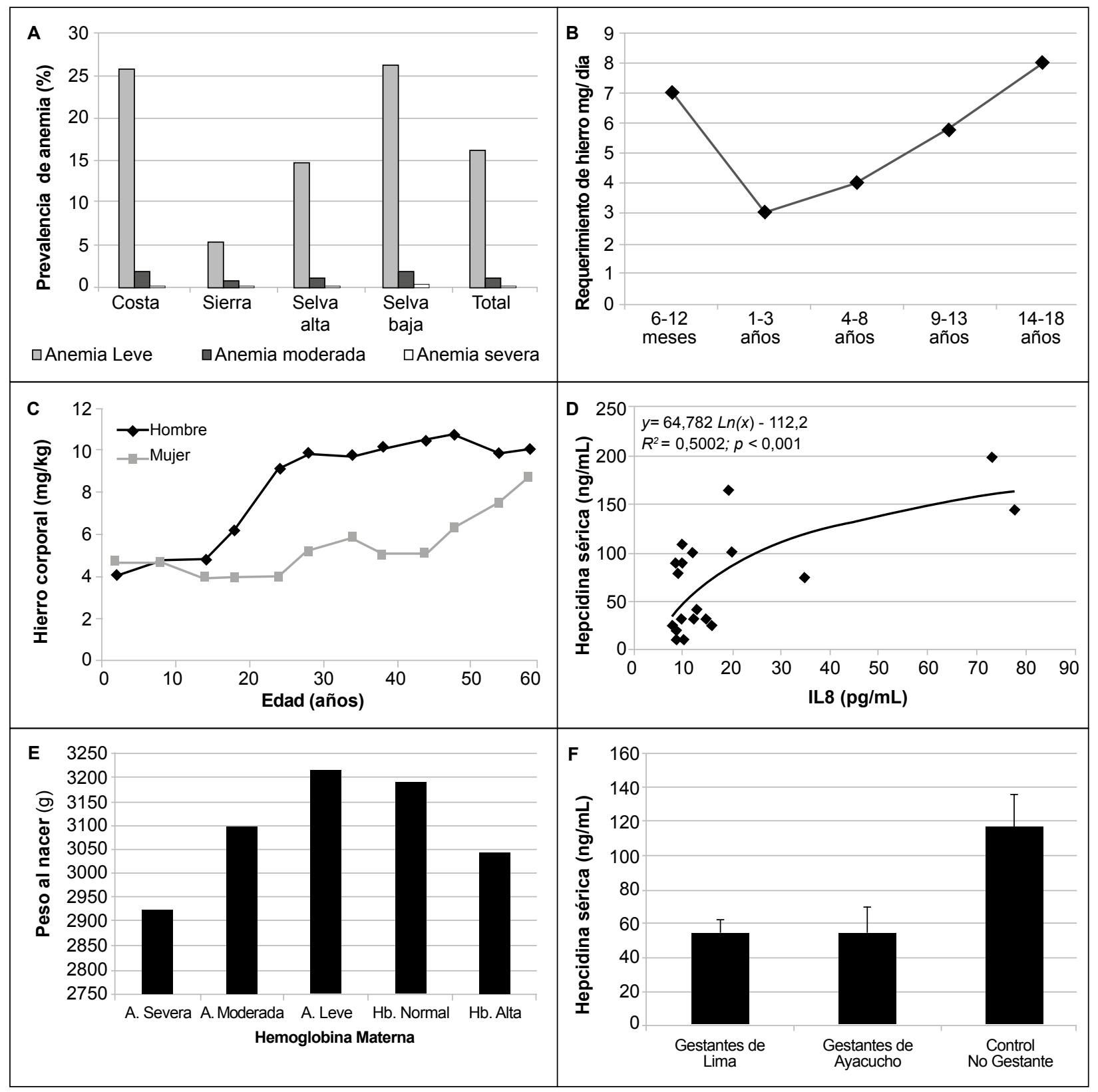

Figura 4. (A) Prevalencia de anemia leve, moderada y severa en las distintas regiones del Perú (2). (B) Requerimientos de hierro (mg/día) desde los 6 meses hasta los 18 años de edad (datos: Food and Nutrition Board, Institute of Medicine, National Academies) ${ }^{(55)}$. (C) Efecto de la edad sobre el contenido de hierro corporal $(\mathrm{mg} / \mathrm{kg})$, todos los valores son positivos e indican la cantidad almacenada de hierro (datos: basados en una muestra de 2057 sujetos colectados en NHANES III) ${ }^{(56)}$. (D) Correlación entre hepcidina sérica (ng/mL) e IL-8 (datos propios). (E) Peso del recién nacido según valor de hemoglobina materna: anemia leve es óptima para peso al nacer. ANOVA: $p<0,01$ (datos propios). (F) Nivel de hepcidina sérica en no gestantes y en gestantes de nivel del mar y de la altura (datos propios). Valores obtenidos en el segundo trimestre de 50 gestantes de nivel del mar y 50 gestantes de la altura. Los niveles de hepcidina fueron medidos usando un kit de ELISA Hepcidin 25 Bioactive (DRG Instruments GmBH. Marburg, Alemania)

\section{DEFINICIÓN DE ANEMIA}

La anemia se define de acuerdo a la edad, sexo, y altitud, basado en consideraciones de distribución estadística y/o por puntos de corte. La anemia en las gestantes y en niños de 6-59 meses se define como valores de hemoglobina $<11$ $\mathrm{g} / \mathrm{dL}$ (1). Teniendo en cuenta que la mayor parte del hierro se encuentra en la hemoglobina, la OMS recomienda su medición para determinar la prevalencia de anemia como una valoración de la deficiencia de hierro (ID) a pesar que ellos mismos reconocen que la anemia no es específica de ID ${ }^{(1)}$.

La anemia materna moderada/severa es la que afecta la salud de la madre y del feto. Las prevalencias de anemia 
materna moderada/severa en hospitales públicos del país son, en promedio, el $2 \%$ de las gestantes estudiadas, con mayor predominancia en la selva (Figura 4A). En tanto, la $\mathrm{Hb}$ alta (>14,5 g/dL) se observa en el $23,7 \%$ de gestantes de la sierra centro y $11,9 \%$ en la sierra sur. Con esto demuestra que en la sierra el problema principal es la eritrocitosis, y esta es mucho menor en los Andes sur que en los centrales ${ }^{(2)}$.

\section{FACTOR DE CORRECCIÓN DE LA HEMOGLOBINA POR LA ALTURA}

La OMS recomienda corregir el punto de corte de la hemoglobina para definir anemia en la altura. La corrección aumenta conforme aumenta la altitud de residencia ${ }^{(1)}$. Esta corrección se basa en la asunción que todas las poblaciones aumentan la hemoglobina conforme aumenta la altura de residencia. Luego de la corrección de la hemoglobina por la altura, la prevalencia de anemia aumenta conforme aumenta la altura ${ }^{(2,32)}$, sugiriendo que estos sujetos diagnosticados como anémicos luego de la corrección de la hemoglobina son deficientes de hierro. En la actualidad se sabe que no es generalizable el aumento de la $\mathrm{Hb}$ con la altura ${ }^{(1,2,8)}$.

En el otro lado de la curva de distribución de la hemoglobina, niveles de $\mathrm{Hb}>14,5 \mathrm{~g} / \mathrm{dL}$ en gestantes, es un factor de riesgo para resultados adversos del embarazo tanto para las madres como para los neonatos ${ }^{(33,34)}$. La eritrocitosis excesiva (EE) se diagnostica cuando los varones tienen

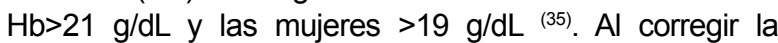
hemoglobina por la altura, aumenta la proporción de sujetos calificados como anémicos, pero, al mismo tiempo, se reduce la proporción de sujetos con eritrocitosis y EE ${ }^{(32)}$. Esto, debido a que la corrección de la $\mathrm{Hb}$ implica mover en la altura la curva de distribución de la $\mathrm{Hb}$ a la izquierda.

Varios estudios han demostrado que la corrección de la hemoglobina por la altura para definir anemia es innecesaria y, por lo tanto, se deberían buscar otros marcadores para evaluar el estado de hierro. Por ejemplo, en Bolivia se ha demostrado que después de corregir la hemoglobina por la altura, la prevalencia de anemia en mujeres adultas es 4,7 veces mayor usando la hemoglobina corregida que usando marcadores de hierro para definir anemia. Igualmente, en niños, la prevalencia de anemia es 3,8 veces mayor usando la hemoglobina corregida que marcadores de hierro ${ }^{(10,32,36)}$.

\section{IMPACTO CLÍNICO DE LA ANEMIA CON HEMOGLOBINA CORREGIDA Y NO CORREGIDA POR LA ALTURA}

Teniendo en cuenta que este factor de corrección es basado en ecuaciones matemáticas, y que con esta corrección la prevalencia de anemia en la altura aumenta de manera importante, se ha analizado el impacto clínico de la anemia, con o sin corrección de la hemoglobina, para determinar si se justifica la corrección de la hemoglobina por altitud.

\section{GESTANTES Y RECIÉN NACIDOS}

En el Perú, la anemia materna leve ( $\mathrm{Hb} 9 \mathrm{a}<11 \mathrm{~g} / \mathrm{dl})$ no tiene impacto negativo sobre el peso del recién nacido, más bien tiene un efecto favorable en la salud materna y del feto ${ }^{(33,34,37,38)}$. Los resultados muestran que la hemoglobina óptima para bajo riesgo de nacer pequeño para edad gestacional se encuentra en el rango definido como anemia leve (9 a <11 g/dL) (Figura 4E). La anemia materna leve a cualquier nivel de altitud no estuvo asociada con riesgo de preeclampsia, ni de hemorragia posparto ${ }^{(33)}$. Esto también ha sido observado en poblaciones de países desarrollados a nivel del mar ${ }^{(39)}$ y en diferentes grupos étnicos ${ }^{(40,41)}$.

Las gestantes con anemia moderada/severa tienen mayor riesgo de muerte fetal tardía, partos pretérminos y de partos de pequeños para edad gestacional, con efectos similares a nivel del mar ${ }^{(42)}$, altitud moderada ${ }^{(33)}$ y gran altitud ${ }^{(34)}$.

Igualmente, los niveles de hemoglobina alta se asocian a efectos adversos, particularmente en el peso del recién nacido $y$, por otro lado, el vivir a moderada o gran altitud tiene un efecto negativo sobre el recién nacido independiente del nivel de hemoglobina, lo cual significa que asociar el efecto de la altura a valores altos de hemoglobina materna es doblemente negativo en la salud del feto ${ }^{(34)}$.

Las diferencias en el crecimiento fetal entre las poblaciones a baja y gran altitud son evidentes a partir de las 33 semanas de embarazo ${ }^{(43)}$, lo que sugiere la necesidad de monitorizar las gestaciones en la altura en este periodo crítico. Esto, debido a las implicancias negativas que tiene en la vida adulta el nacer con bajo peso, pues es conocido que incrementa los riesgos de morbilidad, y mortalidad prematura en la vida adulta. Esto en adición a los conocidos efectos del bajo peso al nacer: incremento del riesgo de muerte fetal tardía, mortalidad perinatal y mortalidad neonatal.

Estos resultados cuestionan la necesidad de dar a todas las gestantes (anémicas y no anémicas) un suplemento de hierro, teniendo en cuenta que valores de hemoglobina por encima de $13,5 \mathrm{~g} / \mathrm{dL}$ se asocian a tasas altas de recién nacidos pequeños para edad gestacional ${ }^{(38)}$, y que confirma trabajos de otros autores que demuestran que mujeres no anémicas viviendo a moderadas altitudes y que recibiendo suplemento de hierro con aumento de la hemoglobina por encima de $14,5 \mathrm{~g} / \mathrm{dL}$, presentan mayores tasas de hijos nacidos pequeños para edad gestacional ${ }^{(44,45,46)}$.

Igualmente, el riesgo de preeclampsia aumenta con valores de hemoglobina materna $>14,5 \mathrm{~g} / \mathrm{dL}$ o $<7,0 \mathrm{~g} / \mathrm{dL}$ y el riesgo de hemorragia posparto aumenta con la anemia moderada/ 
severa. La mortalidad materna se incrementa con la anemia moderada/severa y por la eritrocitosis $(\mathrm{Hb}>14,5 \mathrm{~g} / \mathrm{dL})^{(33)}$.

En gestantes cuya primera medición de hemoglobina indica ausencia de anemia, muestran que aquellas que en la segunda medición presentan $\mathrm{Hb}>14,5 \mathrm{~g} / \mathrm{dL}$, la tasa de nacer pequeño para edad gestacional (SGA) aumenta al doble $(19,9 \%)$ de lo observado en gestantes que se mantienen no anémicas en la segunda medición (10,5\%) ${ }^{(47)}$.

El corregir el punto de corte de la $\mathrm{Hb}$ por la altura, incrementa cinco veces la tasa de anemia; sin embargo, aquellos efectos adversos del recién nacido que se afectan por la anemia como la muerte fetal tardía y el parto pretérmino, más bien se reducen con la corrección del punto de corte de la hemoglobina ${ }^{(11)}$. Esto indicaría que muchas mujeres en la altura calificadas de anémicas por la corrección de la hemoglobina no lo serían realmente, sino que tienen valores más bajos de hemoglobina por estar más bien adaptadas a la altura.

Los hallazgos confirman que las mujeres con valores más bajos de hemoglobina en las alturas del Asia y del Perú tienen mejor resultado reproductivo que aquellas con niveles de hemoglobina más altas ${ }^{(2,5,48)}$.

\section{INFANTES Y NIÑOS MENORES DE 60 MESES}

La menor prevalencia de malnutrición crónica (<2DS del Z-score talla-por-edad) está en el rango de anemia leve, en tanto que la prevalencia de malnutrición crónica aumenta en niños con anemia moderada/severa y en aquellos con $\mathrm{Hb}>14,5 \mathrm{~g} / \mathrm{dL}^{(32)}$.

La prevalencia de niños con estado normal de nutrición se reduce de $82 \%$ en el grupo con hemoglobina normal, a $53 \%$ en el grupo con hemoglobina normal luego de la corrección, debido a que niños con hemoglobina alta y desnutrición crónica están pasando al rango de hemoglobina normal, y niños con hemoglobina normal y nutrición normal están pasando al rango de anémicos.

\section{ADULTOS}

Los adultos con niveles elevados de hemoglobina tienen puntajes más altos para el mal de montaña crónico (MMC). Al ser movida la curva de distribución a la izquierda por corrección de la hemoglobina, el puntaje de MMC aumenta en aquellos que teniendo hemoglobina elevada son calificados de hemoglobina normal luego de la corrección ${ }^{(32)}$.

\section{MARCADORES DEL ESTADO DEL HIERRO}

La justificación de corregir o no la hemoglobina por la altura dependerán de una evaluación del estado de hierro. En la actualidad, los mecanismos para el mantenimiento de la homeostasis de hierro se entienden diferentes a lo que se conocía antes de la era de la hepcidina. La absorción de hierro aumenta cuando el organismo requiere más hierro por ejemplo en la gestación. En ese caso el nivel de hepcidina en suero de la gestante se reduce. Como se ve en la Figura $4 \mathrm{~F}$, el valor de hepcidina es similar en gestantes de nivel del mar y en la altura. Esto indica que la gestante de altura tiene un estado de hierro exactamente igual que a nivel del mar.

Los marcadores del estado de hierro, además de la hepcidina, incluyen a la ferritina sérica (marcador del almacén de hierro en los tejidos), y al receptor soluble de transferrina (marcador de deficiencia de hierro para eritropoyesis). En infantes de 6-24 meses de la ciudad de Puno (3800 m), después de la corrección de la hemoglobina, la prevalencia de anemia de $11,3 \%$ aumenta a $94,7 \%(p<0,01)$. Si valoramos por un marcador de hierro como la ferritina sérica, $26,3 \%$ tienen deficiencia de hierro (ID) (ferritina sérica $<12 \mathrm{ng} / \mathrm{mL}$ ) y $12 \%$ anemia con deficiencia de hierro (IDA; ferritina sérica $<12$ $\mathrm{ng} / \mathrm{mL}$ y $\mathrm{Hb}<11 \mathrm{~g} / \mathrm{dL}$ ).

Después de realizar la corrección de la $\mathrm{Hb}$, el $51,9 \%$ presenta anemia con niveles normales de ferritina, lo cual es inadmisible. Infantes de Puno con niveles de hemoglobina normal ( $\mathrm{Hb}$ : $11-14,5 \mathrm{~g} / \mathrm{dL}$ ) tienen niveles de ferritina sérica mayores que aquellos con anemia leve ( $\mathrm{Hb}: 10,9-10 \mathrm{~g} / \mathrm{dL})$. Al hacer la corrección de la hemoglobina por la altura, los sujetos que pasan a ser diagnosticados como anemia leve tienen niveles normales de ferritina sérica indicando que no son deficientes de hierro y, por lo tanto, están siendo erróneamente clasificados como anémicos ${ }^{(32,49)}$.

En 17703 menores de 60 meses de edad de la región Arequipa, residentes entre 0 y 4500 metros de altitud, la prevalencia de anemia de $16,1 \%$, aumentó a $50 \%$ luego de la corrección de la hemoglobina $(p<0,01)$. Al mismo tiempo, la prevalencia de eritrocitosis se reduce de $3 \%$ a $0,7 \%$ luego de la corrección $(p<0,01)$.

En adultos de 0 a $4340 \mathrm{~m}$ de altitud, la proporción de sujetos con anemia aumenta de $5,5 \%$ a $19,8 \%$ luego de la corrección de la $\mathrm{Hb}(\mathrm{p}<0,01)$, en tanto, la proporción de sujetos con EE se reduce de 4,8 a $0,2 \%(p<0,01)^{(32)}$.

La ferritina sérica y el contenido corporal de hierro en anémicos son menores que en aquellos con anemia luego de la corrección de la $\mathrm{Hb}$, indicando que la corrección aumenta el diagnóstico de personas como anémicos cuando en realidad tienen valores normales de hierro en el organismo.

\section{REFLEXIONES FINALES}

Los estudios en la altura benefician no solo a los diez millones de peruanos que residen sobre los 2000 metros de altitud, sino que también involucra a 44807237 
de latinoamericanos viviendo en las alturas de Bogotá (Colombia), Quito (Ecuador), Puebla, México DF, Estado de México (México), y Cochabamba, Chuquisaca, La Paz, Oruro, y Potosí (Bolivia); y en general a 200 millones de personas que habitan sobre los 2000 metros de altitud en el mundo. Más aun, el presentar niveles de hemoglobina baja $(<9 \mathrm{~g} / \mathrm{dL})$ o alta (>14,5 g/dL) también ocurre a nivel del mar, por lo que el aporte de estos estudios implica a una amplia población mundial.

En países con poblaciones viviendo en la altura, los valores de la hemoglobina corregida son usados para tomar decisiones políticas referentes al cuidado de salud. Más aun, las Guías Técnicas del Ministerio de Salud del Perú, así como en otros países, indican la obligatoriedad de la intervención para prevención o tratamiento con suplementos de hierro en mujeres gestantes, sean o no anémicas, y en niños ${ }^{(50)}$. Particularmente, en poblaciones que viven en la altura estas medidas bien intencionadas pueden exponer a una sobrecarga de hierro, que podría ser más bien perjudicial para la salud. Recientemente, una revisión sistemática da evidencia preclínica de efectos adversos que tiene en la salud cerebral en adultos la ingesta elevada de hierro en neonatos ${ }^{(51)}$.

Estos estudios tienen una alta repercusión en medicina preventiva y salud pública, ya que implica la necesidad de revisar si el suplemento de hierro debe ser universal o solo en casos de anemia moderada/severa. Suplementar con hierro a todas las mujeres gestantes y a niños menores de 5 años, particularmente en la altura, puede aumentar el riesgo de sobrecarga de hierro.

El evitar que un niño nazca con bajo peso monitorizando los niveles de hemoglobina en el embarazo es menos costoso en términos económicos, y más beneficioso en términos de salud que tratar los casos de morbilidad en la vida adulta y que tienen un alto costo para el estado y las familias.
En resumen, la corrección de la hemoglobina por la altura aumenta los casos de anemia que en realidad tienen un contenido de hierro corporal normal. La anemia por deficiencia de hierro (IDA) es un estadio terminal de la deficiencia de hierro (ID). Por ello, cuando empieza a disminuir la reserva de hierro, se debe esperar casos con ferritina sérica disminuida, pero con hemoglobina todavía normal (ID prelatente). La anemia va a desarrollar, cuando la reserva de hierro se agota, la disponibilidad de hierro para la médula ósea conduce a una eritropoyesis con deficiencia de hierro. Este patrón no se observa cuando se usa la corrección de la hemoglobina por la altura. Por el contrario, por acción de la corrección se pueden observar casos de anemia con niveles normales de ferritina sérica.

En conclusión, los nuevos conceptos acerca de la regulación de la homeostasis del hierro, revelan que la recomendación de la OMS de corregir el punto de corte de la hemoglobina para definir anemia en la altura ha llevado en Perú, y probablemente en otros países con poblaciones viviendo en las alturas como los tibetanos y etíopes, a una sobreestimación en las prevalencias de anemia. En la población andina, la corrección de la hemoglobina por la altura favorece un diagnóstico incrementado de anemia, y disminuye la prevalencia de eritrocitosis. Por ello, es necesario usar marcadores apropiados para identificar la deficiencia de hierro en la altura.

Contribuciones de autoría: CVV, DF y GFG han participado en la concepción y diseño del artículo. CVV, DF y GFG han participado en la redacción del artículo. Todos los autores aprobaron la versión final.

\section{Fuentes de financiamiento: autofinanciado}

Conflicto de interés: los autores no tienen conflictos de interés en la publicación de este artículo.

\section{REFERENCIAS BIBLIOGRÁFICAS}

1. Beall CM. Andean, Tibetan, and Ethiopian patterns of adaptation to high-altitude hypoxia. Integr Comp Biol. 2006;46(1):18-24. doi: 10.1093/icb/ icj004.

2. Gonzales GF, Tapia V, Gasco M, Carrillo C. Hemoglobina materna en el Perú: diferencias regionales y su asociación con resultados adversos perinatales. Rev Peru Med Exp Salud Publica. 2011;28(3):484-91.

3. Gonzales GF, Alarcón-Yaquetto DE, Zevallos-Concha A. Human adaptation to life at high altitude. Chapter 8. Human Adaptation to life at high altitude. En: Gelpi RJ et al., (eds). Biochemistry of Oxidative Stress,
Advances in Biochemistry in Health and Disease, Switzerland: Springer International Publishing: 2016; 109-126 p

4. Gonzales GF. Peruvian contributions to the study on human reproduction at high altitude: from the chronicles of the Spanish conquest to the present. Respir Physiol Neurobiol. 2007 Sep 30;158(2-3):172-9.

5. Hartinger S, Tapia V, Carrillo C, Bejarano L, Gonzales GF. Birth weight at high altitudes in Peru. Int J Gynaecol Obstet. 2006;93(3):275-81.

6. Levine LD, Gonzales GF, Tapia VL, Gasco M, Sammel MD, Srinivas SK, et al. Preterm birth risk at high altitude in Peru.
Am J Obstet Gynecol. 2015;212(2):210. e1-8. doi: 10.1016/j.ajog.2014.08.024.

7. Moore LG, Young D, McCullough RE, Droma T, Zamudio S. Tibetan protection from intrauterine growth restriction (IUGR) and reproductive loss at high altitude. Am J Hum Biol. 2001;13(5):635-44.

8. Wu T, Wang X, Wei C, Cheng H, Wang X, Li Y, et al. Hemoglobin levels in Qinghai-Tibet: different effects of gender for Tibetans vs. Han. J Appl Physiol (1985). 2005;98(2):598-604.

9. Soria R, Julian CG, Vargas E, Moore LG, GiussaniDA.Graduated effects ofhigh-al- 
titude hypoxia and highland ancestry on birth size. Pediatr Res. 2013;74(6):633-8. doi: 10.1038/pr.2013.150.

10. Julian CG, Wilson MJ, Lopez M, Yamashiro H, Tellez W, Rodriguez A, et al. Augmented uterine artery blood flow and oxygen delivery protect Andeans from altitude-associated reductions in fetal growth. Am J Physiol Regul Integr Comp Physiol. 2009;296(5):R1564-75. doi: 10.1152/ajpregu.90945.2008

11. Gonzales GF, Tapia V, Carrillo CE. Stillbirth rates in Peruvian populations at high altitude. Int $\mathrm{J}$ Gynaecol $\mathrm{Ob}$ stet. 2008;100(3):221-7.

12. Gonzales GF, Tapia V, Gasco M. Correcting haemoglobin cut-offs to define anaemia in high-altitude pregnant women in Peru reduces adverse perinatal outcomes. Arch Gynecol Obstet. 2014;290(1):6574. doi: $10.1007 /$ s00404-014-3182-z.

13. World Health Organization. Iron Deficiency Anemia. Assessment, Prevention and Control. In A Guide for Progamme Managers. Geneva: World Health Organization; 2001.

14. Reichert CO, da Cunha J, Levy D, Maselli LMF, Bydlowski SP, Spada C. Hepcidin: Homeostasis and Diseases Related to Iron Metabolism. Acta Haematol. 2017;137(4):220-236. doi: $10.1159 / 000471838$.

15. Dev S, Babitt JL. Overview of iron metabolism in health and disease. Hemodial Int. 2017 Jun;21 Suppl 1:S6S20. doi: $10.1111 /$ hdi.12542.

16. Pigeon C, Ilyin G, Courselaud B, Leroyer P, Turlin B, Brissot P, et al. A new mouse liver-specific gene, encoding a protein homologous to human antimicrobial peptide hepcidin, is overexpressed during iron overload. J Biol Chem. 2001;276(11):7811-9.

17. Park CH, Valore EV, Waring AJ, Ganz T. Hepcidin, a urinary antimicrobial peptide synthesized in the liver.J Biol Chem. 2001 Mar 16;276(11):7806-10.

18. Knutson MD. Iron transport proteins: Gateways of cellular and systemic iron homeostasis. J Biol Chem. 2017 Aug 4;292(31):12735-12743. doi: 10.1074/ jbc.R117.786632.

19. Muckenthaler MU, Rivella S, Hentze MW, Galy B. A Red Carpet for Iron Metabolism. Cell. 2017 Jan 26;168(3):344-361. doi: 10.1016/j. cell.2016.12.034.
20. Talbot NP, Lakhal S, Smith TG, Privat C, Nickol AH, Rivera-Ch M, et al. Regulation of hepcidin expression at high altitude. Blood. 2012;119(3):857-60. doi: 10.1182/blood-2011-03-341776.

21. Lundring EL, Janocha AJ, Koch CD, Gebremedhin A, Di Rienzo A, AlkortaAranburu G, et al. Plasma hepcidin of Ethiopian highlanders with steady-state hipoxia. Blood. 2013;122(11):1989-91. doi: 10.1182/blood-2013-03-491068.

22. Talbot NP, Smith TG, Lakhal-Littleton S, Gülsever C, Rivera-Ch M, Dorrington $\mathrm{KL}$, et al. Suppression of plasma hepcidin by venesection during steady-state hypoxia. Blood. 2016;127(9):1206-7. doi: 10.1182/blood-2015-05-647404.

23. Bah A, Pasricha SR, Jallow MW, Sise EA, Wegmuller R, Armitage AE, et al. Serum Hepcidin Concentrations Decline during Pregnancy and May Identify Iron Deficiency: Analysis of a Longitudinal Pregnancy Cohort in The Gambia. J Nutr. 2017;147(6):1131-1137. doi: 10.3945/jn.116.245373.

24. Gao G, Liu SY, Wang HJ, Zhang TW, Yu P, Duan XL, et al.Effects of Pregnancy and Lactation on Iron Metabolism in Rats. Biomed Res Int. 2015;2015:105325. doi: 10.1155/2015/105325.

25. Koenig MD, Tussing-Humphreys L, Day J, Cadwell B, Nemeth E. Hepcidin and iron homeostasis during pregnancy. $\mathrm{Nu}-$ trients. 2014;6(8):3062-83. doi: 10.3390/ nu6083062.

26. Cao C, Fleming MD. The placenta: the forgotten essential organ of iron transport. Nutr Rev. 2016;74(7):421-31.

27. Dahlfors G, Stål P, Hansson EC, Bàràny P, Sisowath C, Onelöv L, et al. Validation of a competitive ELISA assay for the quantification of human serum hepcidin. Scand J Clin Lab Invest. 2015;75(8):6528.

28. Cappellini MD, Comin-Colet J, de Francisco A, Dignass A, Doehner W, S P Lam C, et al. Iron deficiency across chronic inflammatory conditions: International expert opinion on definition, diagnosis, and management. Am J Hematol. 2017;92(10):1068-78.

29. Su J, Li Z, Cui S, Ji L, Geng H, Chai K, et al. The Local HIF-2 $\alpha /$ EPO Pathway in the Bone Marrow is Associated with Excessive Erythrocytosis and the Increase in Bone Marrow Microvessel Density in Chronic Mountain Sickness. High
Alt Med Biol. 2015;16(4):318-30. doi: 10.1089/ham.2015.0015.

30. Sánchez C, Merino C, Figallo M. Simultaneous measurement of plasma volume and cell mass in polycythemia of high altitude. J Appl Physiol, 1970;28(6):775-8.

31. Otto JM, Plumb JO, Clissold E Kumar S, Wakeham DJ, Schmidt W, et al. Hemoglobin concentration, total hemoglobin mass and plasma volume in patients: implications for anemia. Haematologica, 2017;102(9):1477-1485. doi: 10.3324/haematol.2017.169680.

32. Gonzales GF, Rubín de Celis V, Begazo J, Hinojosa MDR, Yucra S, Zevallos $A$, et al. Correcting the cut-off point of hemoglobin at high altitude favors misclassification of anemia, erythrocytosis and excessive erythrocytosis. Am J Hematol. 2017. doi: 10.1002/ajh.24932.

33. Gonzales GF, Tapia V, Gasco M, Carrillo C, Fort AL. Association of hemoglobin values at booking with adverse maternal outcomes among Peruvian populations living at different altitudes. Int $J$ Gynaecol Obstet. 2012 May;117(2):134-9. doi: 10.1016/j.ijgo.2011.11.024.

34. Gonzales GF, Steenland K, Tapia V. Maternal hemoglobin level and fetal outcome at low and high altitudes. Am J Physiol Regul Integr Comp Physiol. 2009 Nov;297(5):R1477-85. doi: 10.1152/ ajpregu.00275.2009.

35. León-Velarde F, Maggiorini M, Reeves JT, Aldashev A, Asmus I, Bernardi L, et al. Consensus statement on chronic and subacute high altitude diseases. High Alt Med Biol. 2005;6(2):147-57.

36. Cook JD, Boy E, Flowers C, Daroca MdelC. The influence of high-altitude living on body iron. Blood 2005;106(4):1441-6.

37. Becerra C, Gonzales GF, Villena A, de la Cruz D, Florián A. Prevalencia de anemia en gestantes, Hospital Regional de Pucallpa, Perú. Rev Panam Salud Publica, 1998;3(5):285-92. Artículo en español.

38. Gonzales GF. Impacto de la altura en el embarazo y en el producto de la gestación. Rev Peru Med Exp Salud Publica. 2012 Jun;29(2):242-9.

39. Steer PJ. Maternal hemoglobin concentration and birth weight. Am J Clin Nutr. 2000;71(5 Suppl):1285S-7S.

40. Steer P, Alam MA, Wadsworth J, Welch A. Relation between maternal haemoglobin concentration and birth weight in different ethnic groups. BMJ. 1995;310(6978):489-91. 
41. Little MP, Brocard P, Elliott P, Steer PJ. Hemoglobin concentration in pregnancy and perinatal mortality: a London-based cohort study. Am J Obstet Gynecol. 2005;193(1):220-6.

42. Kozuki N, Lee AC, Katz J; Child Health Epidemiology Reference Group. Moderate to severe, but not mild, maternal anemia is associated with increased risk of small-forgestational-age outcomes. J Nutr. 2012 Feb;142(2):358-62. doi: 10.3945/ jn.111.149237.

43. Gonzales GF, Tapia V. Birth weight charts for gestational age in 63,620 healthy infants born in Peruvian public hospitals at low and at high altitude. Acta Paediatr. 2009 Mar;98(3):454-8. doi: 10.1111/j.1651-2227.2008.01137.x.

44. Casanueva E, Viteri FE, Mares-Galindo M, Meza-Camacho C, Loría A, Schnaas $\mathrm{L}$, et al. Weekly iron as a safe alternative to daily supplementation for nonanemic pregnant women. Arch Med Res. 2006; 37(5), 674-82.

45. Viteri FE, Casanueva E, Tolentino MC, Díaz-Francés J, Erazo AB. Antenatal iron supplements consumed daily produce oxidative stress in contrast to weekly supplementation in Mexican non-anemic women. Reprod Toxicol. 2012; 34(1): 125-32.

46. Ziaei S, Norrozi M, Faghihzadeh $S$, Jafarbegloo E. A randomised placebo-controlled trial to determine the effect of iron supplementation on pregnancy outcome in pregnant women with haemoglobin $>$ or $=13.2 \mathrm{~g} / \mathrm{dl}$. BJOG. 2007;114(6):684-8.

47. Gonzales GF, Tapia V, Fort AL. Maternal and perinatal outcomes in second hemoglobin measurement in nonanemic women at first booking: effect of altitude of residence in Peru. ISRN Obstet Gynecol. 2012;2012:368571. doi: $10.5402 / 2012 / 368571$

48. Cho JI, Basnyat B, Jeong C, Di Rienzo A, Childs G, Craig SR, et al. Ethnically Tibetan women in Nepal with low hemoglobin concentration have better reproductive outcomes. Evol Med Public Health. 2017 Apr 21;2017(1):8296. doi: 10.1093/emph/eox008.

49. Arosio P, Elia L, Poli M. Ferritin, cellular iron storage and regulation. IUBMB Life. 2017 Jun;69(6):414-422. doi: 10.1002/ iub.1621.

50. Ministerio de Salud. Norma técnica Manejo terapéutico y preventivo de la anemia en niños, adolescentes, mujeres gestantes y puérperas. Lima: Ministerio de Salud; 2017. 1-35 p. Disponible en: http://bvs.minsa.gob.pe/local/ MINSA/4190.pdf

51. Agrawal S, Berggren KL, Marks E, Fox JH. Impact of high iron intake on cognition and neurodegeneration in humans and in animal models: a systematic review. Nutr Rev. 2017 Jun 1;75(6):456-470. doi: 10.1093/nutrit/nux015.

52. Ministerio de Salud, Instituto Nacional de Salud Plan Nacional para la Reducción de la Desnutrición Crónica Infantil y la Prevención de la Anemia en el País, Periodo 2014-2016. Lima: Ministerio de Salud, Instituto Nacional de Salud, 2014 Disponible en: http://www.minsa.gob. pe/portada/especiales/2015/nutriwawa/ directivas/005_plan_reduccion.pdf

53. Bothwell TH. Iron requirements in pregnancy and strategies to meet them. Am J Clin Nutr. 2000;72(1 Suppl):257s$264 s$.

54. Evans P, Cindrova-Davies T, Muttukrishna S, Burton GJ, Porter J, Jauniaux E. Hepcidin and iron species distribution inside the first-trimester human gestational sac. Mol Hum Reprod. 2011 Apr;17(4):227-32. doi: 10.1093/ molehr/gaq101.

55. Grandone A, Marzuillo P, Perrone L, del Giudice EM. Iron metabolism dysregulation and cognitive dysfunction in pediatric obesity: Is there a connection?. Nutrients. 2015 Nov 6;7(11):9163-70. doi: $10.3390 /$ nu7115458.

56. Cook JD, Flowers CH, Skikne BS. The quantitative assessment of body iron. Blood, 2003 May 1;101(9):3359-64.

Correspondencia: Gustavo Francisco Gonzales Rengifo

Dirección: Av. Honorio delgado 430 Urb. Ingeniería San Martin de Porres. Lima, Perú. Teléfono: 01-319-0000 (Anexo: 231332) Correoelectrónico:gustavo.gonzales@upch.pe

\title{
Nuestros artículos se encuentran indizados en:
}

\author{
Publated
}

www.ncbi.nlm.nih.gov/pubmed 